\title{
Effect of Repetitive Transcranial Magnetic Stimulation on Hand Function of Spastic Cerebral Palsy Children
}

\section{Bablu Lal Rajak ${ }^{1}$, Meena Gupta ${ }^{1}$, Dinesh Bhatia ${ }^{1}$ and Arun Mukherjee ${ }^{2}$}

${ }^{1}$ Department of Biomedical Engineering, North Eastern Hill University, Shillong, Meghalaya, India

2 UDAAN-for the Differently Abled, New Delhi, India

"Corresponding author: Bablu Lal Rajak, Department of Biomedical Engineering North Eastern Hill University, Shillong-793022, Meghalaya, India, Tel: 03642307930; E-mail: bablurajak@gmail.com

Received date: December 25, 2016; Accepted date: February 13, 2017; Published date: February 18, 2017

Copyright: @ 2017 Rajak BL, et al. This is an open-access article distributed under the terms of the Creative Commons Attribution License, which permits unrestricted use, distribution, and reproduction in any medium, provided the original author and source are credited.

\begin{abstract}
Repetitive Transcranial magnetic stimulation (rTMS) is emerging as a new investigation as well as treatment tool for various neurological and psychiatric diseases. Recent studies showed its application as treatment tool in movement disorders, where rTMS stimulation on primary motor cortex alters physiological patterns of motor threshold; motor evoked potential and cortical plasticity which induces motor activity. Recent studies on rTMS combined with rehabilitation therapy demonstrated functional improvement in motor activities of spastic cerebral palsy (SCP) children. Thus, this study was designed to evaluate the effect of rTMS on hand function of SCP patients.

Forty-five children diagnosed as SCP participated in this study after written consent from their parents or guardians. They were divided into three groups- control (CG) and interventional group (IG-A and IG-B). Participants in CG were provided only physical therapy (PT) of 30 minutes duration daily for 20 days and those in IG were administered rTMS frequency of $5 \mathrm{~Hz}$ (IG-A) and $10 \mathrm{~Hz}$ (IG-B) for 15 minutes consisting of 1500 pulses daily for 20 days; followed by PT as given to CG. Quality of upper extremity skill test (QUEST) scoring was used for evaluating the improvement in hand function of SCP patients. The pre (before starting any therapy) versus post (after completion of 20 sessions) mean QUEST score between different groups were statistically significant $(p<0.01)$ and the mean change was $0.61,2.46$ and 2.87 in CG, IG-A and IG-B respectively.
\end{abstract}

However, encouraging functional improvement in hand function was observed in diplegic patients in the age group of 2-6 years employing $5 \mathrm{~Hz}$ frequency and higher frequency $(10 \mathrm{~Hz})$ induced better activity in hemiplegic and quadriplegic patient of older age groups (7-16 years).

Keywords: Cerebral palsy; Physical therapy; Quality of upper extremity skill test (QUEST); Transcranial magnetic stimulation

\section{Introduction}

Ever since the introduction of repetitive Transcranial magnetic stimulation (rTMS) in 1989, several studies demonstrated rTMS as an investigational as well as a treatment tool for a variety of neurological and psychiatric disorders [1]. rTMS is a noninvasive brain stimulation technique that repeatedly stimulates cerebral cortex by a train of magnetic pulses. The stimulation modulates cortical excitability producing physiological changes in motor threshold; motor evoked potential and cortical plasticity [2]. These physiological changes can induce motor activity and helps in the treatment of movement disorders.

The application of rTMS in movement disorders was thoroughly reviewed by Kamble et al., where diseases such as Parkinson's and Huntington's disease, dystonia, progressive supranuclear palsy, etc., were discussed [3]. Additionally, recent publications reported positive effect of rTMS in cerebral palsy [4] demonstrating improved motor activity [5,6] along with reduction in muscle tightness [7].

Cerebral Palsy (CP) is a neurodevelopmental disorder that affects a developing child. $\mathrm{CP}$ occurs due to brain damage occurring in the fetal period or infancy which results in motor or sensory nerve damage leading to inability to perform activities of daily living [8]. CP presents itself in different forms - ataxic, spastic and dyskinetic; among which spastic CP is most common. Spastic cerebral palsy is a neuromuscular impairment that limits the movement and posture of the body due to increased tonic stretch reflex or exaggerated tendon reflex in the muscles [9]. These patients are not able to perform coordinated motor activities of upper and lower extremities.

Besides having difficulty in performing hand function such as grasping, lifting and weight bearing. In order to restore this motor disorder, diverse therapeutic approaches [10] are being employed, commonly known are task oriented training [11] and physical therapy [12]. These physical approaches helps in functional organization by repeatedly training on activity tasks associated with daily living based on motor learning and promotes controlled movements that are actually used when performing functional tasks [13]. Thus, in this study, we aimed to evaluate the effect of rTMS on hand function of spastic CP children combined with physical training (PT) exercises.

\section{Materials and Methods}

\section{Participants}

Forty-five participants diagnosed as spastic CP by consultant physician and pediatric neurologists participated in this study after 
written consent from their parents or guardians. These participants were equally divided into three groups namely, control group (CG), interventional group A (IG-A) and interventional group B (IG-B) matching age, $\mathrm{CP}$ type and age group. The demographic characteristic of participants is given in Table 1. Only participants that satisfied our inclusion criteria were selected from the out-patient department of UDAAN- for the differently abled, Delhi a non-profit organization that pioneered the rehabilitation of $\mathrm{CP}$ children using various interventions. The inclusion criteria followed was willingness to participate; age group between 2 to 16 years; muscle tightness mild to moderate, cognitive deficiency nil to moderate, limited hand functiongrasping or weight bearing, no metallic implants, no uncontrolled seizures or congenital diseases. Data of some spastic CP children (3 in RG and 2 each in IG-A and IG-B) that showed near to normal hand function were not included for any analysis.

\begin{tabular}{|l|l|l|l|}
\hline Variables & CG & IG-A & IG-B \\
\hline Age \pm SD (Years) & $8.59 \pm 4.81$ & $8.33 \pm 4.33$ & $7.24 \pm 5.01$ \\
\hline Height \pm SD $(\mathrm{cm})$ & $107.00 \pm 24.80$ & $114.71 \pm 26.93$ & $118.17 \pm 15.99$ \\
\hline Weight + SD $(\mathrm{kg})$ & $21.58 \pm 15.62$ & $27.14 \pm 10.50$ & $25.67 \pm 13.85$ \\
\hline Sex & \multicolumn{3}{|l}{} \\
\hline Male : Female & $7: 5$ & $8: 5$ & $9: 4$ \\
\hline
\end{tabular}

CP type

\begin{tabular}{|l|l|l|l|}
\hline Hemiplegic & 4 & 3 & 3 \\
\hline Diplegic & 5 & 7 & 6 \\
\hline Quadriplegic & 3 & 3 & 4 \\
\hline
\end{tabular}

\section{Age group}

\begin{tabular}{|l|l|l|l|}
\hline $2-6$ years & 4 & 5 & 5 \\
\hline $7-11$ years & 4 & 6 & 5 \\
\hline $12-16$ years & 4 & 2 & 3 \\
\hline
\end{tabular}

Table 1: Demographic characteristic of participants.

\section{Stimulation device}

TMS device used in this study delivered repetitive trains of magnetic pulses using Neuro-MS/D Variant-2 therapeutic (Neurosoft, Russia) with angulated figure of eight shaped coil (AFEC-02-100-C). The device had two channel Neuro-EMG-MS digital system for determining the motor threshold. The eight-shaped coil generated magnetic field of up to 4 Tesla at the center of the coil that when placed on the skull penetrates the cranium and enters into the soft tissue of the brain which stimulates the motor neurons. In this study, the coil was placed on the primary motor cortex which is known to be the motor pathway signaling center of the brain.

\section{Assessment tool}

The assessment tool used in this study was quality of upper extremity skill test (QUEST) which is a specially developed tool to overcome the limitation of measures of hand function in children with motor disabilities [14]. QUEST scoring is universally used to evaluate the functions of upper extremity that demands fine motor skills $[15,16]$. QUEST comprises of descriptive and impairment based measures which is designed to evaluate the hand function in spastic CP.

QUEST evaluates 36 items of upper extremity in four domains, namely, A- dissociated movements, B- grasping, C- protective extension and D- weight bearing. In order to calculate QUEST score, total values of each domain are added and averaged, then converted to percentage which was used for analysis.

\section{Research design}

The selection of participants and design of study protocol was performed only after approval from the institutional ethics committee for human samples or participants (IECHSP) of the host institution. Immediately after selection of participants, pre assessment of QUEST was performed on all participants of different groups namely, CG, IGA and IG-B. Participants of CG were provided only PT for 30 minutes daily for 20 days (5 days per week for 4 weeks) whereas children in IGA was administered rTMS of $5 \mathrm{~Hz}$ frequency and those in IG-B with $10 \mathrm{~Hz}$ comprising of 1500 pulses (50 pulses per train with total 30 trains having inter-train delay of 20 seconds) for 15 minutes daily for 20 days.

The rTMS session of both the groups were followed by PT of 30 minutes as provided to participants in RG. After completion of 20 sessions of different therapies administered to different groups, postassessment of QUEST was performed. Both PT and rTMS sessions were administered by trained professionals and the assessment was done by a trained physiotherapist who was kept blinded to the research protocols used in the study.

\section{Statistical analysis}

The pre and post QUEST scores for each of the three groups were analyzed with a paired-sample $t$-test to determine statistical significance. The variance and covariance analyses were also performed. The mean and median QUEST scores were used to evaluate the percentage of functional gain that was induced by different therapies in different groups. Moreover, the percent change in QUEST scores were used to determine improvement in different spastic CP types and age groups. All statistical analysis was performed using SPSS 20.0 (Armonk, NY, IBM Corp., USA) and Microsoft Excel 2010. The $p$-value of less that 0.01 was considered statistically significant.

\section{Results}

The paired $t$-test between the pre and post QUEST scores in control (only physical therapy) group (CG) revealed significant differences ( $t=$ -3.368, $d f=11, p=0.006$, confidence interval [CI] -1.0003 to -0.2097 ) with mean change of $0.61 \%$. The $t$-test between pre-post assessments of QUEST scores in interventional (rTMS+PT) groups was also significant. In IG-A, $\mathrm{t}=-4.036, \mathrm{df}=12, \mathrm{p}=0.002, \mathrm{CI}-3.789$ to -1.133 and in IG-B, $\mathrm{t}=-3.768, \mathrm{df}=12, \mathrm{p}=0.003, \mathrm{CI}-4.439$ to -1.186 . The mean change score was $2.46 \%$ in IG-A and $2.82 \%$ in IG-B. The QUEST scores between different groups are represented in Table 2. 
Citation: Rajak BL, Gupta M, Bhatia D, Mukherjee A (2017) Effect of Repetitive Transcranial Magnetic Stimulation on Hand Function of Spastic

Page 3 of 5

\begin{tabular}{|l|l|l|l|l|l|l|l|l|}
\hline \multirow{2}{*}{ Groups } & \multicolumn{3}{l}{ Min } & \multicolumn{2}{l}{ Max } & \multicolumn{2}{l|}{ Median } & \multicolumn{2}{l|}{ Mean \pm SD } \\
\cline { 2 - 10 } & Pre & Post & Pre & Post & Pre & Post & Pre & Post \\
\hline CG & 39.76 & 40.97 & 88.75 & 90.23 & 63.84 & 64.68 & $62.79 \pm 15.40$ & $63.40 \pm 15.42$ \\
\hline IG-A & 50.93 & 50.93 & 98.21 & 100 & 76.44 & 82.93 & $75.61 \pm 15.89$ & $78.07 \pm 16.24$ \\
\hline IG-B & 49.29 & 51.61 & 91.67 & 98.15 & 80.22 & 81.71 & $74.28 \pm 15.22$ & $77.10 \pm 14.87$ \\
\hline
\end{tabular}

Table 2: Descriptive statistics of control and interventional groups.

The improvement (mean change) in different groups was $0.61,2.46$ and 2.82 percent in CG, IG-A and IG-B respectively (Figure 1). This clearly demonstrated positive effect of rTMS over PT. But it was observed that mean change in both the interventional groups differed slightly; IG-B with $10 \mathrm{~Hz}$ rTMS frequency revealed better improvement than IG-A $(5 \mathrm{~Hz})$. However, considering the change in the median score (Figure 2), $5 \mathrm{~Hz}$ seemed better that $10 \mathrm{~Hz}$ frequency. This lead to ambiguity in the interpretation of result, thus in order to overcome this ambiguity, percentage functional gain according to CP type and age group was analyzed.

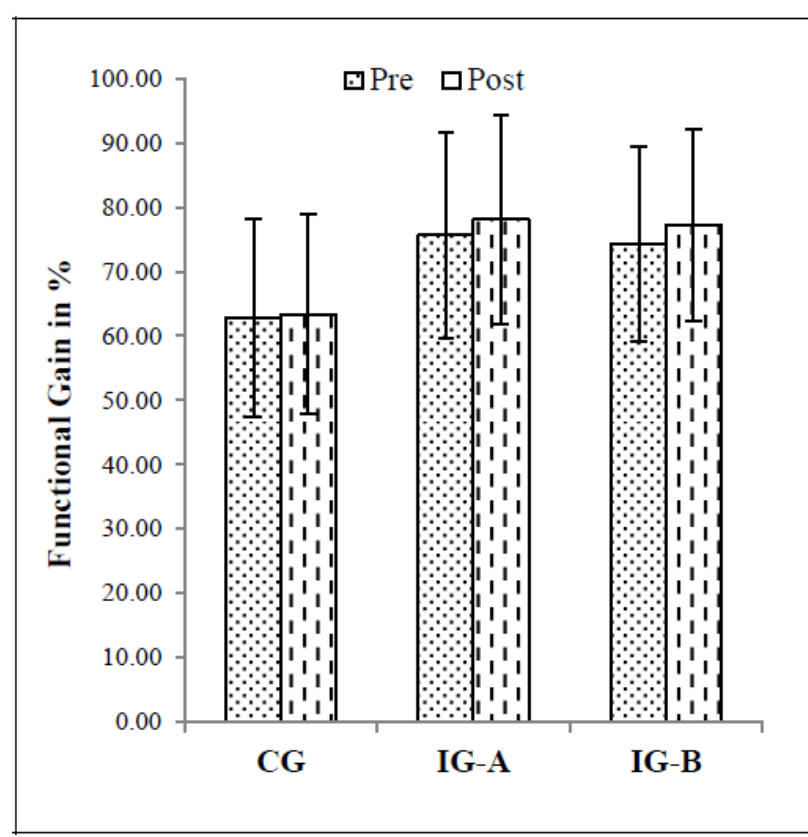

Figure 1: Functional improvement percentage in range of functional gain in QUEST score between QUEST scores between different groups.

The mean change between different spastic CP types (hemiplegic, diplegic and quadriplegic) in various groups showed minimal improvement by only PT (CG) compared to rTMS therapy group (IG$A$ and IG-B). Between IG-A and IG-B, $10 \mathrm{~Hz}$ frequency showed appreciable functional gain in hand function in hemiplegic $(4.04 \%)$ and quadriplegic (3.87\%) patients as compared to diplegic $(2.31 \%)$ patients. On the contrary, $5 \mathrm{~Hz}$ rTMS frequency was more beneficial in diplegic $(2.31 \%)$ patients compared to hemiplegics or quadriplegics in the same group (Figure 3).

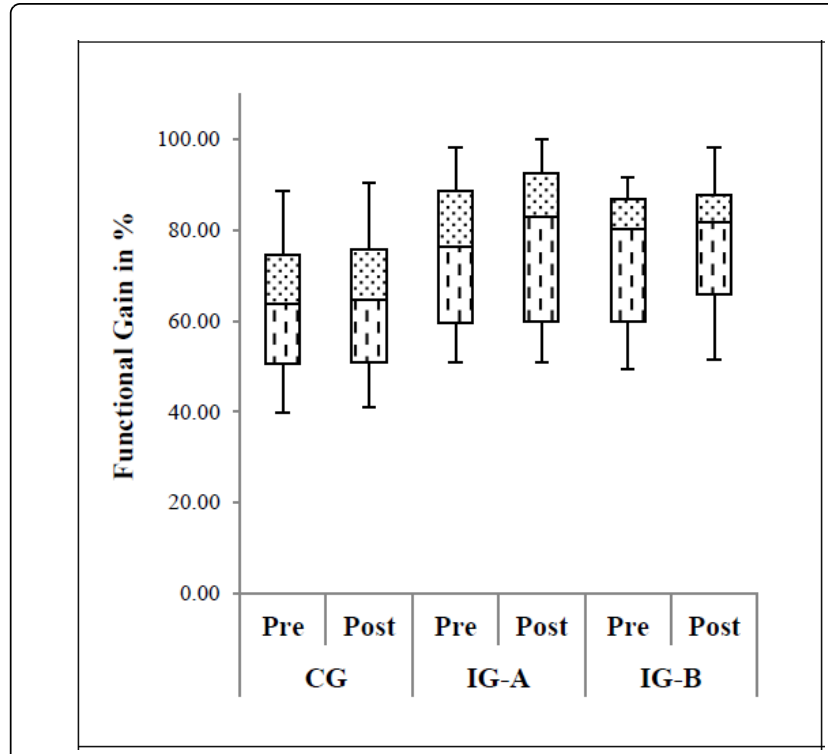

Figure 2: Box-plot showing change in median.

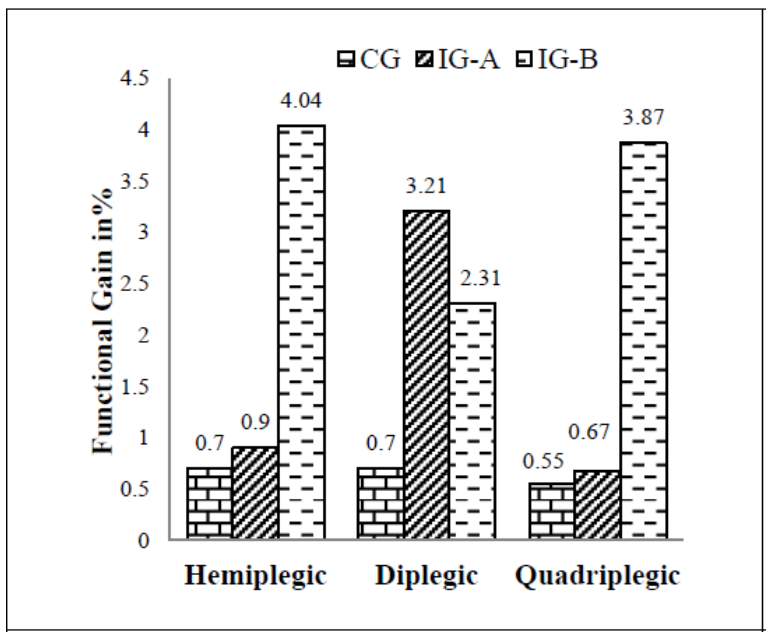

Figure 3: Improvement percentage between different spastic CP types following different therapy regime.

Comparing the effect of different therapies between different age groups, it was observed that combined rTMS and PT (IG) group demonstrated better functional hand activity compared to only PT 
(CG). rTMS frequency of $10 \mathrm{~Hz}$ induced better improvement $(4.14 \%)$ in older children (12-16 y) compared to lower age group (2-11 y) children but $5 \mathrm{~Hz}$ frequency was more beneficial $(3.7 \%)$ in younger children (2-6 y) compared to older spastic CP patients (Figure 4).

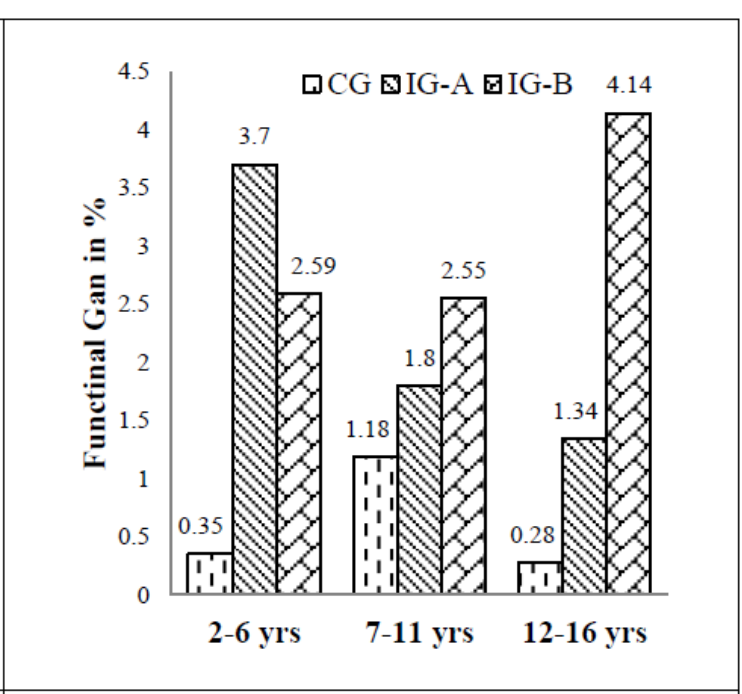

Figure 4: Improvement percentage between different age groups following different therapy regime.

The result demonstrated that though rTMS induces appreciable functional gain in hand function when compared to the traditionally employed physical training, lower frequency $(5 \mathrm{~Hz})$ lead to better improvement in diplegic CP patients that are in the age group of 2-6 years and higher frequency $(10 \mathrm{~Hz})$ induced better functional improvement in hemiplegic and quadriplegic patients of older age group in limited number of sessions.

\section{Discussion and Conclusion}

The interventions employed in the restoration of functional abilities in $\mathrm{CP}$ patients to make them perform activities of daily living are diverse and emerging. This diversity in interventions existed since there is no specific diagnostic protocol, nor is there a clear singular etiology and pathology for CP. Now-a-days rehabilitation of these patients is performed employing technology such as robotics, virtual reality and brain stimulations [17]. All these interventions are known to induce neural plasticity which traditional approaches fail to meet. rTMS too, induces brain plasticity [18] and produces lasting changes in brain function with potential therapeutic effects $[19,20]$. Studies on spinal cord injury (SCI), multiple sclerosis and stroke patients provide good evidence to show the effectiveness of rTMS combined with rehabilitation therapy on motor function [21-23]. Similarly, our findings in this study demonstrated that high frequency rTMS is beneficial in spastic CP cases to enhance their functional hand activity; but the effect of rTMS frequency differed accordingly between different $\mathrm{CP}$ types and age group of patients.

\section{Acknowledgment}

This work is supported by funding received (Ref: SEED/TIDE/ 007/2013) from the Technology Intervention for Disabled and Elderly of the Department of Science and Technology (DST), Government of
India, New Delhi. The authors also acknowledge the support of all the staffs of UDAAN-for the differently abled, Delhi. The authors are grateful to the children and their parents for participating in this sponsored study.

\section{References}

1. Daskalakis ZJ, Christensen BK, Fitzgerald PB, Chen R (2002) Transcranial magnetic stimulation: A new investigational and treatment tool in psychiatry. J Neuropsychiatry Clin Neurosci 14: 406-415.

2. Pascual-Leone A, Valls-Sole J, Wassermann EM, Hallet M (1994) Responses to rapid rate transcranial magnetic stimulation of the human motor cortex. Brain 117: 847e58.

3. Kamble N, Netravathi M, Pal PK (2014) Therapeutic applications of repetitive transcranial magnetic stimulation (rTMS) in movement disorders: A review. Parkinsonism Relat Disord 20: 695-707.

4. Ramezani S, Amini N, Sadeghi N, Safakheil H, Vousooghi N (2016) Brain stimulation techniques in cerebral palsy. J Pediatr Neurol Med 1:2.

5. Gupta M, Rajak BL, Bhatia D, Mukherjee A (2016) Transcranial magnetic stimulation therapy in spastic cerebral palsy children improves motor activity. J Neuroinfect Dis 7: 231.

6. Feng J, Du L, Shan L, Wang B, Li H (2015) Infra-low frequency transcranial magnetic stimulation effectively improves the motor function in children with spastic cerebral palsy. J Neurol Neurophysiol 6: 2.

7. Gupta M, Rajak BL, Bhatia D, Mukherjee A (2016) Effect of r-TMS over standard therapy in decreasing muscle tone of spastic cerebral palsy patients. Journal of Medical Engineering \& Technology 40: 210-216.

8. Bax M, Goldstein M, Rosenbaum P, Leviton A, Paneth N, et al. (2005) Proposed definition and classification of cerebral palsy. Devl Med Child Neurol 47: 571-576.

9. Morris C (2007) Definition and classification of cerebral palsy: a historical perspective. Developmental Medicine \& Child Neurology 49: 3-7.

10. Shierk A, Lake A, Haas T (2016) Review of therapeutic interventions for the upper limb classified by manual ability in children with cerebral palsy. In: Seminars in plastic surgery 30: 14-23.

11. Kim JY, Kim JM, Ko EY (2014) The effect of the action observation physical training on the upper extremity function in children with cerebral palsy. J Exerc Rehabil 10: 176-183.

12. Piazza B, Hennrikus W, Schell R, Armstrong D, Fortuna K (2016) The factors most important for quality of life in children and adolescents with cerebral palsy. J Pediatr Neurol Med 1:102.

13. Wu C, Trombly CA, Lin K, Tickle-Degnen L (2000) A kinematic study of contextual effects on reaching performance in persons with and without stroke: Influences of object availability. Arch Phys Med Rehabil.81: 95-101.

14. DeMatteo C, Law M, Russell D, Pollock N, Rosenbaum P, et al. (1993) The reliability and validity of the quality of upper extremity skills test. Physical \& Occupational Therapy in Pediatrics 13: 1-18.

15. Naylor CE, Bower E (2005) Modified constraint-induced movement therapy for young children with hemiplegic cerebral palsy: A pilot study. Developmental Medicine \& Child Neurology 47: 365-369.

16. Sharma R, Sinha AG (2016) Impact of physiotherapy service utilization on physical and functional status of children with cerebral palsy in Jalandhar, Punjab, India. CHRISMED Journal of Health and Research 3: 173.

17. Gaebler-Spira D, Peri E, Lunardini F, Sanchez-Santed F, Duff M, et al. (2016) Rehabilitation technologies for cerebral palsy. Emerging Therapies in Neurorehabilitation II 10: 87-108.

18. Wu SW, Gilbert DL (2015) Measuring neuroplasticity in children using brain stimulation. Developmental Medicine \& Child Neurology 57: 499.

19. Pascual-Leone A, Tormos JM, Keenan J, Tarazona F, Cañete C, et al. (1998) Study and modulation of human cortical excitability with transcranial magnetic stimulation. J Clin Neurophysiol 15: 333-343. 
Citation: Rajak BL, Gupta M, Bhatia D, Mukherjee A (2017) Effect of Repetitive Transcranial Magnetic Stimulation on Hand Function of Spastic Cerebral Palsy Children. J Neurol Disord 5: 329. doi:10.4172/2329-6895.1000329

Page 5 of 5

20. Khedr EM, Ahmed MA, Fathy N, Rothwell JC (2005) Therapeutic trial of repetitive transcranial magnetic stimulation after acute ischemic stroke. Neurology 65: 466-468.

21. Elkholy SH, Atteya AA, Hassan WA, Sharaf M, Gohary AME (2014) Low rate repetitive transcranial magnetic stimulation (rtms) and gait rehabilitation after stroke. Int J Neurorehabilitation 1: 2376-0281.

22. Mally J, Dinya E (2008) Recovery of motor disability and spasticity in post-stroke after repetitive transcranial magnetic stimulation (rTMS) Brain research bulletin 76: 388-395.
23. Kumru H, Benito J, Murillo N, Valls-Sole J, Valles M, et al. (2013) Effects of high-frequency repetitive transcranial magnetic stimulation on motor and gait improvement in incomplete spinal cord injury patients. Neurorehabil Neural Repair 27: 421-429. 